Psychiatric comorbidity was high (49\%), of which generalised anxiety disorder was the most common (59\%).

Referrals were typically from primary care $(77 \%)$. Approximately half $(51 \%)$ had reported suicidal thoughts according to the referral.

A quarter of patients $(26 \%)$ were seen by CMHT within 8 weeks of referral; $20 \%$ of referrals however waited over 12 months before being assessed.

Risk assessments were out of date for $71 \%$ of patients.

$100 \%$ of patients had a crisis plan noted within their most recent clinic letter; however, none of these met the required standards.

Polypharmacy was common (60\%), with $34 \%$ prescribed two antidepressants. Use of lithium augmentation was uncommon, with only one patient prescribed this. $43 \%$ were prescribed an antipsychotic; of which, $29 \%$ had appropriate physical health monitoring completed.

Over half of patients (60\%) had been referred to psychology services; of these, $38 \%$ had either completed or were in ongoing treatment at the time of review.

Conclusion. CMHTs manage the care of individuals with depression who have high levels of active symptoms and disability, psychiatric comorbidity, care requirements, and complex treatment plans. Pharmacological management was broadly in line with guidelines, and rates of referral to psychology were satisfactory. Risk assessment and crisis planning are clear areas in need of urgent attention in order to comply with guidelines and ensure patient safety.

\section{Epidemiology and current treatment patterns of treatment-resistant depression in Scotland: a CPRD study}

Timothy Ming ${ }^{2 \star}$, Tom Denee ${ }^{1}$, Gemma Scott ${ }^{1}$, Joachim Morrens ${ }^{2}$ and Christopher Weatherburn ${ }^{3}$

${ }^{1}$ Janssen-Cilag Ltd; ${ }^{2}$ Janssen Cilag and ${ }^{3}$ Dundee Health and Social Care Partnership

${ }^{\star}$ Corresponding author.

doi: 10.1192/bjo.2021.876

Aims. To assess the incidence and treatments currently used in clinical practice for the treatment of treatment-resistant depression (TRD) in Scotland.

Background. Patients with major depressive disorder (MDD) who have not responded to at least two successive antidepressant (AD) treatments in a single episode are described as having TreatmentResistant Depression (TRD). Epidemiological data on TRD in Scotland is lacking. Furthermore, there is no data to our knowledge on therapies prescribed in Scottish clinical practice to treat TRD.

Method. A retrospective, longitudinal cohort study was conducted using Clinical Practice Research Datalink (CPRD) medical records. Adult patients were indexed on $\mathrm{AD}$ prescription, requiring MDD diagnosis within 90 days, from Jan 2011-May 2018 with 360-day baseline and 180-day minimum follow-up periods. Failure of $\geq 2$ adequate oral AD regimens following indexing constituted TRD classification. Incidence rates of MDD and TRD (within the MDD cohort) and treatment lines following TRD classification were derived.

Result. The analysis included 20,059 patients with MDD (mean age 44 years, $63 \%$ female, median follow-up 59 months); 1,374 (6.8\%) were classified as TRD. Median time-to-TRD classification was 25 months. The incidence rate of MDD was 15.9 per 1,000 patient-years and for TRD was 14.7 per 1,000 MDD-patientyears. For all first four post-TRD treatment lines, SSRI monotherapy was the most commonly prescribed therapy, followed by combination (dual/triple) therapy and augmentation therapy (at least one oral $\mathrm{AD}$ supplemented with lithium, an antipsychotic or an anticonvulsant therapy). At first-line of TRD treatment, $1,050(76.4 \%)$ patients received monotherapy AD, 212 (15.4\%) received combination $\mathrm{AD}$ therapy and $112(8.2 \%)$ received augmentation therapy. The most common monotherapy treatments at first-line TRD were sertraline (15.6\%), mirtazapine (13.8\%), fluoxetine (12.2\%) and venlafaxine (11.6\%). Among combination therapies, mirtazapine, venlafaxine, sertraline and amitriptyline were frequently used. Among the TRD and MDD cohort, no somatic treatments were coded in CPRD, although the use of these treatments was likely underestimated.

Conclusion. Monotherapy AD treatment was the most common therapy type for all four post-TRD treatment lines. These data support the need for new treatments that can achieve and maintain therapeutic response, and avoid continuous cycling through similar AD therapies.

This study was sponsored by Janssen Cilag Ltd.

\section{COVID-19 and young people with intellectual} disability: a service review

Omer Minhas ${ }^{1 \star}$, Pippa Mundy ${ }^{2}$ and Jessica Stewart ${ }^{2}$

${ }^{1}$ Cwm Taf Morgannwg University Health Board and ${ }^{2}$ Cardiff and

Vale University Health Board

${ }^{*}$ Corresponding author.

doi: 10.1192/bjo.2021.877

Aims. A service review of specialist child and adolescent intellectual disability provision in South Wales was conducted between March and May 2020. The purpose was to explore the impact of the first COVID-19 pandemic lockdown on children with intellectual disability and their families. The review aimed to explore if the disruption to the systems involved in their care would impact their wellbeing and behavioural presentations. We also measured if there had been an increase in the use of medication. Our focus was on the distress calls, which are requests for urgent clinical review to prevent a crisis.A service review of specialist child and adolescent intellectual disability provision in South Wales was conducted between March and May 2020. The purpose was to explore the impact of the first COVID-19 pandemic lockdown on children with intellectual disability and their families. The review aimed to explore if the disruption to the systems involved in their care would impact their wellbeing and behavioural presentations. We also measured if there had been an increase in the use of medication. Our focus was on the distress calls, which are requests for urgent clinical review to prevent a crisis.

Method. Six clinical areas across three Welsh health boards under the same specialist team were surveyed. Case notes and email correspondence were reviewed to obtain the number and content of crisis calls made to specialist CAMHS across an eight week period during the first UK COVID-19 lockdown. Data were gathered on frequency, purpose, and outcome of calls. Comparison data were also collected for the period October 2019 to March 2020.

Result. Pre-COVID-19: Two crisis calls were identified in two different areas during the pre-COVID period surveyed. Increases in medication and increases in respite care packages were made as a result.

During COVID-19 restrictions: 20 different initial distress calls made (children age 9 and 17 years old $(M=13.2) ; 75 \%$ were boys) across five of the six clinical areas. Of these 20 calls, 17 were active cases and 3 were new referrals. $95 \%$ of calls resulted in medication increases and there were few other interventions used due to COVID-19 constraints. Changes to the child's support system were discussed across all cases and return to school was highlighted as 
a key protective factor in improved well-being. Differences between clinical areas were also identified.

Conclusion. There was a clear increase in distress calls and requests to prescribe or increase psychotropic medication to calm the distress during the 'lockdown'. Changes in behavioural presentation may have occurred partly due to the disruption to the complex systems that typically support a child and the shift away from community support. Children with intellectual disability and their families are unique and embedded in complex systems comprising schools, respite, and healthcare provision which work together to deliver optimal mental healthcare with psychosocial interventions with medication for higher-risk situations. Any shifts in these systems may lead to an imbalance and a higher likelihood of medication use.

\section{Audit into post diagnostic support in newly diagnosed dementia patients}

Madhumanti Mitra* and Raghupathy Paranthaman

Greater Manchester Mental Health NHS Foundation Trust ${ }^{*}$ Corresponding author.

doi: 10.1192/bjo.2021.878

Aims. This audit aims to identify whether newly diagnosed dementia patients are offered post diagnostic support and potential factors influencing patient choice.

Background. A diagnosis of dementia can be life changing and hence post-diagnostic support for dementia is key. Multiple guidelines suggest that post diagnostic support need to be offered to all patients diagnosed with dementia. The Department of Health and Social Care and other national/ local guidelines suggest that post diagnostic support is offered to all patients diagnosed with dementia.

Method. Data were collected for 40 patients diagnosed with dementia. Using random number generator, patient group was selected from pool of patients diagnosed with dementia between July' 2017 - December' 2017. Data included whether they had been offered support during the initial appointment and what post-diagnostic support was offered. Demographic details obtained to identify patterns of support accessed by patients.

Result. All patients were offered post-diagnostic support. Diagnosis was discussed in appointment in about $93 \%$ of patients. Medication was discussed in $82 \%$ patients. Driving was discussed in only $64 \%$ patients and LPA was discussed in only $63 \%$ patients. When given choice between Post diagnostic support group (PDSG) and Dementia adviser (DA), slightly more women tend to choose PDSG group. The only 2 ethnic minority patients chose DA. $21 \%$ more patients opted for PDSG group when they had a carer. Conclusion. The positive is that some post-diagnostic support is offered to all patients. Although discussion of diagnosis with patients was done well, discussion of medication, driving and LPA can be improved upon. Ethnicity and family structure/ carer may have a bearing on patient choice of post-diagnostic support.

\section{Medication charts and consent to treatment documentation audit in an acute mixed in-patient psychiatry unit in south Manchester}

Madhumanti Mitra*, Shahid Hussain, Emma Raynor, Joanna Wong and Jennifer Thom

Greater Manchester Mental Health NHS Foundation Trust ${ }^{\star}$ Corresponding author.

doi: 10.1192/bjo.2021.879
Aims. The main aim of this audit was to look at documentation in medication charts in an acute mixed inpatient unit in South Manchester. In addition, we also looked at completion of capacity assessment and consent to treatment forms as appropriate.

Background. Safe prescription, administration and monitoring of medication is key to effective patient care. Due to the busy nature of inpatient hospital wards, errors do unfortunately occur both with the medications, and with the recording of their administration.

We will use a data collection tool to collect data as per standards described in our local GMMH policy. The medication chart will be used as the standard, as this is the current chart that is in use in the Trust.

Method. Data were collected from 31 medication charts for inpatients admitted in the ward between the $5 / 12 / 19$ to $18 / 12 / 19$. We captured data from each page of the medication chart that required a record to be made by any staff, including details of prescribing, administration and pharmacist checks. Data were recorded as either Yes/No or NA (Not Applicable). Data were then summarised and analysed using MS excel.

Result. Of the 31 patients, $22(71 \%)$ had a capacity assessment form completed and 16 (52\%) had a consent to treatment form completed. From the data analysis, it was clear that there are high rates of completion for the 'essential' parts of all prescriptions, including medicine name, dose, route and data. 'Route' was only recorded for $40 \%$ of prescriptions for depot medicines. Details of the administration of a medicine by a nurse was generally well-completed. For as required medications, all information relating to administration (date, time, dose and given by) were fully completed for $100 \%$ of prescriptions. For regular prescriptions however, the administration details were not as wellcompleted, where date of administration was recorded in $84 \%$ of prescriptions and signature in $29 \%$ of prescriptions. Unique patient identifiers are well-recorded on Page 1 of the prescription chart, though not maintained throughout the prescription chart. Nature of reaction to an allergy or sensitivity was only recorded in 6 of the 21 patients (29\%).

Conclusion. Overall, there were good completion rates for the mandatory parts of the prescriptions. However improvements could be made for prescriptions as well as administration and pharmacy checks. The capacity assessment and consent to treatment forms could be improved upon too. We plan to put the recommendations and re-audit in 3-6 months' time.

Service evaluation and research project: pros and cons of centralisation of ECT services in Pennine Care NHS Foundation Trust

Madhumanti Mitra* and Katherine Hayden

Pennine Care NHS Foundation Trust

${ }^{\star}$ Corresponding author.

doi: 10.1192/bjo.2021.880

Aims. The aim of this project would be to understand about the pros and cons/ benefits and risks of using a centralised model of ECT (currently being followed due to COVID restrictions) rather than a decentralised model of ECT (which has been the norm for a long time). A comparative account of both types of systems would help identify whether it could be implemented in Pennine Care and the results could be potentially transferable to other similar settings.

Method. An online survey was undertaken from staff members working in Pennine Care who have been involved in ECT delivery and management. The survey was undertaken between 07/12/ 